feeding. Science. 2004;304(5667):108-110.

6. Bouret SG, Gorski JN, Patterson CM, Chen S, Levin BE, Simerly RB. Hypothalamic neural projections are permanently disrupted in diet-induced obese rats. Cell Metab. 2008;7(2):179-185.

7. Dietrich MO, Horvath TL. Synaptic plasticity of feeding circuits: hormones and hysteresis. Cell. 2011; 146(6):863-865

8. Sun K, Kusminski CM, Scherer PE. Adipose tissue remodeling and obesity. J Clin Invest. 2011; 121(6):2094-2101.

9. Kaushik S, et al. Autophagy in hypothalamic AgRP neurons regulates food intake and energy balance. Cell Metab. 2011;14(2):173-183.

10. Lumeng CN, Saltiel AR. Inflammatory links between obesity and metabolic disease. J Clin Invest. 2011; 121(6):2111-2117.

11. Meng Q, Cai D. Defective hypothalamic autophagy directs the central pathogenesis of obesity via the IkappaB kinase beta (IKKbeta)/NF-kappaB pathway. J Biol Chem. 2011;286(37):32324-32332.

12. McNay DEG, Briançon N, Kokoeva MV, MaratosFlier E, Flier JS. Remodeling of the arcuate nucleus energy-balance circuit is inhibited in obese mice. J Clin Invest. 2012;122(1):142-152.

13. Thaler JP, et al. Obesity is associated with hypothalamic injury in rodents and humans. J Clin Invest. 2012;122(1):153-162.

14. Ettinger MP, et al. Recombinant variant of ciliary neurotrophic factor for weight loss in obese adults: a randomized, dose-ranging study. JAMA. 2003;289(14):1826-1832.

15. Watt MJ, et al. CNTF reverses obesity-induced insulin resistance by activating skeletal muscle AMPK. Nature Med. 2006;12(5):541-548.

16. Kokoeva MV, Yin H, Flier JS. Neurogenesis in the hypothalamus of adult mice: potential role in energy balance. Science. 2005;310(5748):679-683.

17. Kokoeva MV, Yin H, Flier JS. Evidence for constitutive neural cell proliferation in the adult murine hypothalamus. J Comp Neurol. 2007; 505(2):209-220.

18. Shanley LJ, Irving AJ, Rae MG, Ashford ML, Harvey J. Leptin inhibits rat hippocampal neurons via activation of large conductance calcium-activated K+ channels. Nat Neurosci. 2002;5(4):299-300.

19. Purkayastha S, Zhang G, Cai D. Uncoupling the mechanisms of obesity and hypertension by targeting hypothalamic IKK-beta and NF-kappaB. Nat Med. 2011;17(7):883-887.

20. Freeman JL, et al. MR imaging and spectroscopic study of epileptogenic hypothalamic hamartomas: analysis of 72 cases. AJNR Am J Neuroradiol. 2004; 25(3):450-462.

\title{
Rare serotype adenoviral vectors for HIV vaccine development
}

\author{
Nelson L. Michael
}

US Military HIV Research Program, Walter Reed Army Institute of Research, Bethesda, Maryland, USA.

\begin{abstract}
Human adenoviral vectors are being developed for use in candidate vaccines for HIV-1 and other pathogens. However, this approach suffered a setback when an HIV-1 vaccine using an adenovirus type 5 (Ad5) vector failed to reduce, and might even have increased, the rate of HIV infection in men who were uncircumcised and who had preexisting antibodies specific for Ad5. This increased interest in the evaluation of serologically distinct adenoviral vectors. In this issue of the JCI, Frahm and coworkers report evidence that preexisting cellular immune responses directed toward Ad5 reduce the immunogenicity of antigens expressed in Ad5-vectored vaccines and have cross-reacting potential with non-Ad5 adenoviral vectors. The implications of this observation need to be carefully evaluated in future clinical trials of all serotypes of adenovirus-vectored vaccines.
\end{abstract}

The joint United Nations Program on HIV/ AIDS (UNAIDS) estimates that more than 33 million people were living with HIV at the end of 2009 (1). As witnessed by a series of watershed results in the HIV prevention field of late, some progress has been made toward tackling this pandemic. Adult male circumcision (2-4), antiretroviral-based vaginal microbicides (5), preexposure prophylaxis (6), and antiretroviral therapy as a means of prevention (7) have shown varying degrees of efficacy, from modest to potent. These results have together energized the HIV prevention field and have provided a growing number of tools that could be brought to bear in global HIV disease control. However, as all of these approaches

Conflict of interest: The author has declared that no conflict of interest exists.

Citation for this article: J Clin Invest. 2012; 122(1):25-27. doi:10.1172/JCI60988. pose significant challenges for deployment as public health tools, a preventive HIV vaccine, development of which has thus far been elusive despite intensive research efforts, remains a critical goal (8).

\section{HIV vaccine approaches}

There are three main types of vaccine being developed for the prevention of HIV infection: subunit vaccines, recombinant virus-vectored vaccines, and DNA vaccines. Three of the most significant clinical trials conducted thus far tested the efficacy of different combinations of the first two types of vaccine (9-11). When the first vaccine type was tested in two phase III clinical trials, it was disappointingly found that vaccination with repeated doses of gp120 protein subunit vaccines (AIDSVAX B/B' and $\mathrm{B} / \mathrm{E}$ ) failed to protect either men who have sex with men (MSM) (10) or injection drug users (11) from HIV infection. Further disappointment came in the form of the results of the phase III Step study (9), which showed that three injections of a Merckdeveloped adenovirus serotype 5 (Ad5) vector containing gag/pol/nef HIV-1 gene inserts (referred to as the MRKAd5/HIV-1 vaccine) also failed to protect MSM. In contrast to these results, the RV144 trial demonstrated that an HIV vaccine might be possible (12). The approach tested in this trial was to first prime participants with four injections of a canarypox vector containing gag/pro/gp120/ gp41 HIV-1 gene inserts (ALVAC-HIV) and then boost with two injections of a gp120 protein subunit vaccine (AIDSVAX-B/E), and it reduced the rate of HIV infection in a low-incidence population of Thais with heterosexual HIV transmission risk.

The Step study raised the added concern that, following vaccination, uncircumcised MSM with naturally acquired immunity to Ad5, in the guise of preexisting Ad5-specific neutralizing antibodies (nAbs), appeared to experience a transient period of increased risk of infection with HIV (13). These concerns led to the cancellation of a planned phase IIB clinical trial of a related Ad5-vectored gag/pol/env vaccine, which was to be used together with a DNA vaccine prime, in diverse risk groups and the generation of a new, smaller efficacy study focusing on circumcised MSM with no serologic evidence of previous Ad5 exposure (14). Additional research has suggested that the immunoge- 


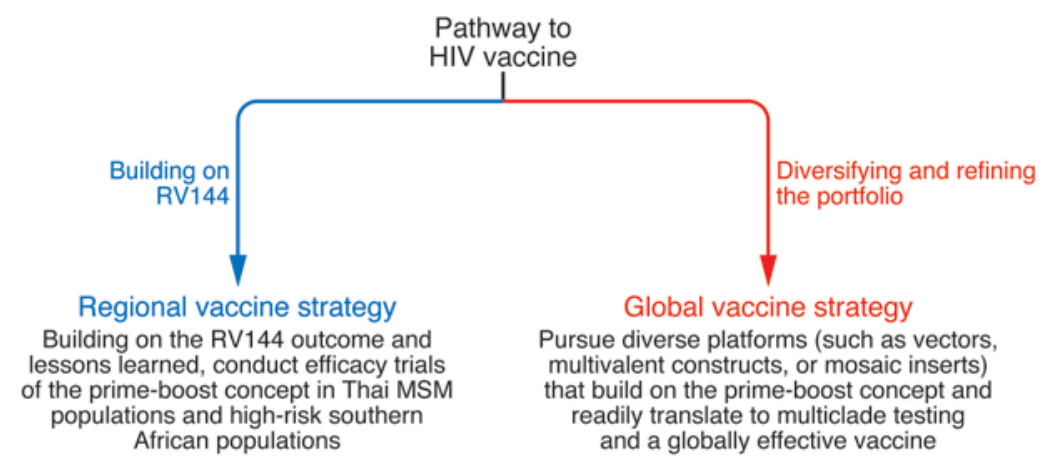

Figure 1

A framework for HIV vaccine development. Two parallel pathways in an HIV vaccine development framework can be envisaged. One pathway (which leads to a regional vaccine strategy) builds on the poxvirus prime protein-subunit boost vaccine concept tested in the RV144 clinical trial (12). Such vaccines would test the ability of additional boosts and adjuvant formulations to improve the durability of protection observed in RV144 and evaluate performance in new risk and geographic populations. The second pathway (which would lead to a global vaccine strategy) seeks to evaluate universal or globally effective HIV vaccines using novel HIV vaccine types (including rare serotype human adenoviral vectors), insert design, adjuvants, and clinical study designs.

nicity of the proteins encoded by the genes inserted into the Ad5 vector was reduced by preexisting Ad5-specific nAbs (15).

The failure of the MRKAd5/HIV-1 vaccine to provide protection from infection with HIV and the concerns it raised regarding preexisting Ad5-specific nAbs was a severe setback, but it did not eliminate all interest in using adenoviral vectors in candidate vaccines, as these platforms are highly attractive in terms of their marked immunogenicity, low cost, and production efficiency. However, the Step results did spur interest in the development of adenoviral vectors from both human and nonhuman primate adenoviruses with a lower global prevalence of preexisting nAbs so-called rare serotype adenoviral vectors as substitutes for Ad5. Rich debate continues on predicting the utility of these rare serotype adenoviral vectors based on typespecific $\mathrm{nAb}$ prevalence $(16,17)$.

\section{Cross-reacting cell-mediated immune responses}

In this issue of the JCI, Frahm et al. examine the impact of evoked cellular immune responses to Ad5 on subsequent immunogenicity to HIV-specific inserts expressed in human volunteers (18), which has been less studied than humoral responses. This is important because cellular immune epitopes may be more broadly shared among adenovirus serotypes than humoral epitopes. Using samples largely from the Step study, the authors asked whether measures of cellular immune reactivity to the Ad5 vector have implications for the immunogenicity of the insert-encoded HIV proteins. Vaccine-evoked adenovirus-specific $\mathrm{CD}^{+} \mathrm{T}$ cell responses were found to correlate indirectly with both the magnitude of the $\mathrm{CD}^{+} \mathrm{T}$ cell responses directed toward the insert-encoded HIV proteins and the breadth of $\mathrm{CD}^{+} \mathrm{T}$ cell responses to these antigens. Importantly, these associations were not influenced by preexisting Ad5-specific nAbs. As some of the adenoviral cellular epitopes were shown to be prevalent across disparate adenovirus types, the data generated by Frahm and colleagues raise concerns about the suitability of rare serotype adenoviruses (e.g., Ad26 and Ad35) for vaccine development in populations with prevalent Ad5-reactive $\mathrm{CD} 4^{+} \mathrm{T}$ cells.

\section{HIV vaccine clinical development strategies}

Prime boost vaccination strategies using rare serotype human adenoviral vectors have shown much promise (19). Rare serotype adenoviral vectors are in various stages of vaccine development for malaria, tuberculosis, hepatitis, and filoviruses. However, could the cross-reacting adenovirus-specific $\mathrm{T}$ cell responses detected by Frahm and colleagues (18) limit the potency of vaccines based on rare serotype human adenoviruses? Would this indicate the intrinsic unfeasibility of developing rare serotype human adenovirus-vectored vaccines? Will we, rather, need to turn to other alternative vectors derived from non-human primates (20), or should adenoviral vector approaches remain an active sector of vaccine development?

These are critical questions to answer, as the field of HIV vaccine development is now at a crossroads. Do we pursue the promising pathway leading from the RV 144 clinical trial (12) and further develop poxvirus gp120 prime boost strategies in different risk populations and in different geographical populations exposed to distinct circulating HIV subtypes? While the advantages of building on success are clear, there are obstacles to this pathway. Notably, as the gp120 products used in the poxvirus prime and the protein subunit boost are inherently specific to a defined HIV-1 subtype (subtype CRF01_AE in the poxvirus prime and CRF01_AE and B for the protein boost), they represent a challenge for the development of a globally effective vaccine. Alternatively, do we explore newer classes of HIV vaccine candidates with more universal subtype application using mosaic HIV gene designs (21) or related technologies?

The likely answer is suggested by the adage "When you come to a fork in the road, take it" (Figure 1). A product development focus would follow up RV144 by testing related products in vaccine efficacy studies in new risk groups, such as MSM in Thailand exposed to HIV subtype CRF01_AE and men and women in southern Africa exposed to subtype $\mathrm{C}$. The other pathway stemming from the fork would explore global strategies using novel vector and insert combinations that could possibly work for all HIV-1 subtypes. The use of heterologous rare serotype human adenoviral vectors would fall into this category of research exploration (14). The promise of a globally effective vaccine derived from this research is highly attractive. The study of correlates of risk of infection and, ultimately, correlates of protection afforded by vaccines of this type is likely to be highly informative for understanding immunopathogenesis, immunogenetics, and viral biology given the diversity of populations and vaccine types tested in this framework.

\section{The way forward}

The impressive volume of cellular immunology data generated through the well executed studies of Frahm and colleagues (18) provide further evidence of the value of human immunology for the development of vaccines against HIV and other infectious pathogens. Will preexisting cellular immunity to Ad5 have a mitigating 
impact on the immunogenicity of HIV antigens vectored in rare serotype human adenoviral vectors? Frahm et al. did not have samples from such clinical trials available to them to test this hypothesis. However, clinical trials employing these vectors have started, and more will follow soon, which will allow for the hypothesis to be tested in the context of rigorous clinical investigation, thus providing critical insight to guide the development of a globally effective HIV vaccine.

\section{Acknowledgments}

The views expressed here belong solely to the author and are not to be construed as reflecting the views of the Department of the Army or the Department of Defense.

Address correspondence to: Nelson L. Michael, US Military HIV Research Program, Walter Reed Army Institute of Research, 6720-B Rockledge Drive, Suite 400, Bethesda, Maryland 20817, USA. Phone: 301.500.3601; Fax: 301.500.3666; E-mail: nmichael@hivresearch.org.

1. UNAIDS. Global Report: UNAIDS Report of the Global AIDS Epidemic. Geneva, Switzerland: Joint United Nations Program on HIV/AIDS (UNAIDS); 2010. 2. Auvert B, Taljaard D, Lagarde E, Sobngwi-Tam- bekou J, Sitta R, Puren A. Randomized, controlled intervention trial of male circumcision for reduction of HIV infection risk: the ANRS 1265 Trial. PLoS Med. 2005;2(11):e298.

3. Bailey RC, et al. Male circumcision for HIV prevention in young men in Kisumu, Kenya: a randomised controlled trial. Lancet. 2007; 369(9562):643-656.

4. Gray RH, et al. The impact of male circumcision on HIV incidence and cost per infection prevented: a stochastic simulation model from Rakai, Uganda. AIDS. 2007;21(7):845-850.

5. Abdool Karim Q, et al. Effectiveness and safety of tenofovir gel, an antiretroviral microbicide, for the prevention of HIV infection in women. Science. 2010;329(5996):1168-1174.

6. Grant RM, et al. Preexposure chemoprophylaxis for HIV prevention in men who have sex with men. N Engl J Med. 2010;363(27):2587-2599.

7. Cohen MS, et al. Prevention of HIV-1 infection with early antiretroviral therapy. $N$ Engl J Med. 2011;365(6):493-505.

8. Johnston MI, Fauci AS. HIV vaccine developmentimproving on natural immunity. NEnglJ Med. 2011; 365(10):873-875.

9. Buchbinder SP, et al. Efficacy assessment of a cellmediated immunity HIV-1 vaccine (the Step Study): a double-blind, randomised, placebo-controlled, testof-concept trial. Lancet. 2008;372(9653):1881-1893.

10. Flynn NM, Forthal DN, Harro CD, Judson FN, Mayer KH, Para MF. Placebo-controlled phase 3 trial of a recombinant glycoprotein 120 vaccine to prevent HIV-1 infection. J Infect Dis. 2005; 191(5):654-665.

11. Pitisuttithum P, et al. Randomized, double-blind, placebo-controlled efficacy trial of a bivalent recombinant glycoprotein $120 \mathrm{HIV}-1$ vaccine among injection drug users in Bangkok, Thailand. J Infect Dis. 2006;194(12):1661-1671.
12. Rerks-Ngarm S, et al. Vaccination with ALVAC and AIDSVAX to prevent HIV-1 infection in Thailand. N Engl J Med. 2009;361(23):2209-2220.

13. Duerr AC, Huang Y, Moodie Z, Lawrence D, Robertson D, Buchbinder S. Analysis of the relative risk of HIV acquisition among Step study participants with extended follow-up. Presented at: AIDS Vaccine Conference; September 28-October 1, 2010; Atlanta, Georgia, USA.

14. Kim JH, Rerks-Ngarm S, Excler JL, Michael NL. HIV vaccines: lessons learned and the way forward. Curr Opin HIV AIDS. 2010;5(5):428-434.

15. Cheng $C$, et al. Differential specificity and immunogenicity of adenovirus type 5 neutralizing antibodies elicited by natural infection or immunization. J Virol. 2010;84(1):630-638.

16. Abbink P, et al. Comparative seroprevalence and immunogenicity of six rare serotype recombinant adenovirus vaccine vectors from subgroups $B$ and $D$. J Virol. 2007;81(9):4654-4663.

17. Mast TC, et al. International epidemiology of human pre-existing adenovirus (Ad) type-5, type-6, type- 26 and type-36 neutralizing antibodies: correlates of high Ad5 titers and implications for potential HIV vaccine trials. Vaccine. 2010;28(4):950-957.

18. Frahm N, et al. Human adenovirus-specific $T$ cells modulate HIV-specific T cell responses to an Ad5-vectored HIV-1 vaccine. J Clin Invest. 2012; 122(1):359-367.

19. Liu J, et al. Immune control of an SIV challenge by a T-cell-based vaccine in rhesus monkeys. Nature. 2009; 457(7225):87-91.

20. Farina SF, et al. Replication-defective vector based on a chimpanzee adenovirus. J Virol. 2001; 75(23):11603-11613.

21. Kong WP, et al. Expanded breadth of the T-cell response to mosaic human immunodeficiency virus type 1 envelope DNA vaccination.J Virol. 2009; 83(5):2201-2215

\title{
IL-1 and atherosclerosis: a murine twist to an evolving human story
}

\author{
Daniel J. Rader
}

Department of Medicine and Institute for Translational Medicine and Therapeutics, Perelman School of Medicine at the University of Pennsylvania, Philadelphia, Pennsylvania, USA.

\begin{abstract}
Inflammation is a critical component of atherosclerosis. IL-1 is a classic proinflammatory cytokine that has been linked to atherosclerosis. A clinical trial has been launched in which an antibody specific for IL-1 $\beta$ is being studied for its effects on cardiovascular events in patients with atherosclerosis. In this issue of the JCI, Alexander et al. report that mice lacking the receptor for IL-1 unexpectedly have features of advanced atherosclerosis that suggest the atherosclerotic plaques may be less stable. These findings illustrate the complexity of inflammatory pathways in atherosclerosis and suggest the need for careful calibration of antiinflammatory approaches to atherosclerosis.
\end{abstract}

Coronary artery disease (CAD) is the leading cause of death in the United States for both men and women. CAD is caused by

Conflict of interest: Daniel J. Rader declares a conflict of interest with Aegerion, Merck, Novartis, and Pfizer.

Citation for this article: J Clin Invest. 2012;

122(1):27-30. doi:10.1172/JCI61163. the progressive formation in the coronary arteries of atherosclerotic plaques that are characterized by the accumulation of lipids, in particular cholesterol and its derivatives, and inflammatory cells. In the early stages of disease development, the atherosclerotic plaques are small, lipid rich, and asymptomatic. Over time, they mature into advanced atherosclerotic plaques, with increased content of vascular smooth muscle cells, extracellular matrix, and inflammatory markers, and gain characteristics that may have clinical consequences. Clinical symptoms can arise from plaques causing flow-limiting stenoses, although the process of compensatory remodeling involving expansion of the vessel can help to protect against this. The more clinically important consequence of coronary atherosclerosis is the rupture or disruption of a "vulnerable" plaque. Plaque rupture leads to prothrombotic material in the plaque becoming exposed to the blood and to thrombosis, resulting in acute coronary events, including myocardial infarction and death. There has been substantial 